\title{
ON THE FIXED POINTS OF SYLOW SUBGROUPS OF TRANSITIVE PERMUTATION GROUPS: CORRIGENDUM
}

\author{
MARCEL HERZOG and CHERYL E. PRAEGER
}

(Received 30 August 1977)

\begin{abstract}
The proof of Theorem 5 in a paper with the same title is incorrect. In this note weaker versions of that theorem are proved.
\end{abstract}

Subject classification (Amer. Math. Soc. (MOS) 1970): 20 B 05.

In Herzog and Praeger (1976) we stated Theorem 5 which is incorrect for $p>2$. Theorem 1 and Corollaries 2-4 are unaffected, as well as Lemmas 2.1 and 2.2. It follows from Praeger (1978b) and Theorem 1 that Corollary 7 is true.

Using the results of Praeger (1978b) we shall prove the following weaker version of Theorem 5 .

THEOREM 5'. Let $G$ be a transitive permutation group on a set $\Omega$ of $n$ points, and let $P$ be a Sylow p-subgroup of $G$ for some prime $p$ dividing $|G|$. Suppose that $P$ has $t$ long orbits and $f$ fixed points in $\Omega$, and suppose that $f=t p-1$. If $P$ has an orbit of length $p$, then $t=1, n=2 p-1$ and $G \supseteq A_{n}$.

Proof. By Praeger (1978a) it follows that all long orbits of $P$ have the same length, namely $p$. Hence $f=t p-1=\frac{1}{2}(n-1)$, and by Praeger (1978b) $t=1$, $n=2 p-1$ and $G \supseteq A_{n}$.

Finally we shall show that Theorem 5 holds for $p=2$ and $f>0$.

THEOREM $5^{\prime \prime}$. Let $G$ be a transitive permutation group on a set $\Omega$ of $n$ points, and let $S$ be a nontrivial Sylow 2-subgroup of $G$. Suppose that $S$ has $t$ long orbits and $f$ fixed points in $\Omega$, and suppose that $f=2 t-i_{2}(n)>0$. Then $t=f=i_{2}(n)=1$ and $G$ is 2-transitive. If the long $S$-orbit has length 2 , then $n=3$ and $G \cong S_{3}$. 
Proof. If $n \leqslant 3$, then Theorem $5 "$ clearly holds. Assume, by induction, that the result is true for transitive groups of degree less than $n$. By Wielandt (1964) 3.7, $|N(S): S|$ is divisible by $f=2 t-i_{2}(n)$. Since $|N(S): S|$ is odd, $f$ is odd, and hence $i_{2}(n)=1$.

Let $\Sigma=\left\{B_{1}, \ldots, B_{r}\right\}$ be a set of blocks of imprimitivity for $G$ in $\Omega$. Since $f>0$ and since $S$ fixes setwise any block containing a point of $\operatorname{fix}_{\Omega} S$, it follows that fix $_{\Sigma} S$ is non-empty. Let $B \in \mathrm{fix}_{\Sigma} S$ and set $f_{B}=\left|\mathrm{fix}_{B} S\right|, f_{\Sigma}=\left|\mathrm{fix}_{\Sigma} S\right|$. Denote by $t_{B}$ and $t_{\Sigma}$ the number of long $S$-orbits in $B$ and $\Sigma$, respectively. Suppose first that $S$ acts nontrivially on $B$. Then by Herzog and Praeger (1976) Theorem $1, f_{B}=2 t_{B}-d$ for some $d \geqslant 1$. Hence by Herzog and Praeger (1976), Lemma 1.2,

$$
2 t-1=f=f_{\Sigma} f_{B}=2 f_{\Sigma} t_{B}-f_{\Sigma} d \leqslant 2 t-f_{\Sigma} d
$$

as $f_{\Sigma} t_{B}$ is the number of long $S$-orbits in $U\left\{B \mid B \in \mathrm{fix}_{\Sigma} S\right\}$. Therefore $f_{\Sigma}=d=1$ and $t_{B}=f_{\Sigma} t_{B}=t$, from which we conclude that $|\Sigma|=1$. On the other hand, if $S$ acts trivially on $B$, then by Herzog and Praeger (1976), Lemma 1.2 and Theorem 1,

$$
f=|B| f_{\Sigma}, \quad t=|B| t_{\Sigma} \quad \text { and } f_{\Sigma}=2 t_{\Sigma}-d
$$

for some $d \geqslant 1$. Hence $2 t-1=f=2 t-|B| d$ and so $|B|=1$. Thus $G$ is primitive on $\Omega$.

Let $\alpha \in \mathrm{fix}_{\Omega} S$ and let $\Gamma_{1}, \ldots, \Gamma_{r}, r \geqslant 1$, be the orbits of $G_{\alpha}$ on $\Omega-\{\alpha\}$. By Wielandt (1964), 18.4, $S$ acts nontrivially on each $\Gamma_{i}$. Let $S$ have $f_{i}$ fixed points and $t_{i}$ long orbits in $\Gamma_{i}$ for $1 \leqslant i \leqslant r$. Then by Herzog and Praeger (1976), Theorem $1, f_{i}=2 t_{i}-d_{i}$ for some $d_{i} \geqslant 1,1 \leqslant i \leqslant r$, and so

$$
2 t-1=f=1+\Sigma f_{i}=1+\Sigma\left(2 t_{i}-d_{i}\right)=2 t+1-\Sigma d_{i},
$$

that is, $\Sigma d_{i}=2$. If $r>1$, then $r=2$ and $d_{1}=d_{2}=1$. By induction $G_{\alpha}$ is 2-transitive on $\Gamma_{1}$ and $\Gamma_{2}$, a contradiction to Wielandt (1964), 17.7. Hence $r=1$, that is $G$ is 2-transitive. If $f>1$, then by Wielandt (1964), 3.7 applied to $G$ and to $G_{\alpha},|N(S): S|$ is divisible by the even integer $f(f-1)$, a contradiction. Hence $f=1$ and so $t=1$. Finally, if $S$ has an orbit of length 2 , then $n=3$ and $G \cong S_{3}$.

\section{References}

M. Herzog and C. E. Praeger (1976), 'On the fixed points of Sylow subgroups of transitive permutation groups', J. Austral. Math. Soc. (Ser. A) 21, 428-437.

C. E. Praeger (1978a), 'Sylow subgroups of transitive permutation groups, II' (to appear).

C. E. Praeger (1978b), 'On transitive permutation groups with a subgroup satisfying a certain conjugacy condition' (submitted).

H. Wielandt (1964), Finite permutation groups (New York-London, Academic Press, 1964).

Department of Mathematics

Institute of Advanced Studies

Australian National University

Canberra, Australia 2600
Department of Mathematics

University of Western Australia

Nedlands, WA, Australia 